\title{
Cinacalcet and Acute Heart Failure on Dialysis - A New Association
}

\author{
Suzanne H Forbes ${ }^{\mathrm{a}, \mathrm{c}}$, Dhakshinamurthy Vijay Anand ${ }^{\mathrm{a}}$, \\ R. Andrew Archibold ${ }^{\mathrm{b}}$, Neil Ashman ${ }^{\mathrm{a}}$
}

\begin{abstract}
Secondary hyperparathyroidism (SHPT) is common in end stage renal disease and is associated with abnormal bone and mineral metabolism, vascular calcification, bone fracture and increased cardiovascular risk. Cinacalcet is an increasingly widely-used calcimimetic agent for the control of SHPT in such patients and trial data has confirmed its use as an efficacious strategy in achieving target parathyroid hormone (PTH) targets. Here we describe a previously undocumented side-effect of cinacalcet treatment in a man on haemodialysis who developed a new and severe dilated cardiomyopathy in clear association with starting cinacalcet. This subsequently resolved fully after withdrawal of the drug. This case, we believe, is the first documented case of severe left ventricular systolic dysfunction temporally associated with cinacalcet use.
\end{abstract}

Keywords: Cinacalcet; Secondary hyperparathyroidism; Dilated cardiomyopathy; Left ventricular systolic dysfunction; Haemodialysis

\section{Introduction}

Secondary hyperparathyroidism is a frequent and often difficult to treat problem encountered in managing patients with end stage kidney disease. Cinacalcet is used in the treatment of secondary hyperparathyroidism in such patients, and in selected patients with primary hyperparathyroidism. It is variably tolerated and has several well documented side effects, the most common of which is gastrointestinal upset. Here, however, we present a unique case and novel asso-

\footnotetext{
Manuscript accepted for publication January 2, 2013

${ }^{\text {a}}$ Royal London Hospital, UK

${ }^{\mathrm{b}}$ London Chest Hospital, UK

${ }^{\mathrm{c} C}$ Corresponding author: Suzanne H Forbes, Renal Unit, Royal London

Hospital, Whitechapel Road, London E1 1BB, UK.

Email: Suzanne.Forbes@bartshealth.nhs.uk

doi: http://dx.doi.org/10.4021/jmc1029w
}

ciation of a serious clinical consequence of this increasingly used drug. We describe an acute dilated cardiomypathy associated with cinacalcet use in a haemodialysis patient, which fully reversed on withdrawal of the drug and for which no other cause was established.

We feel this is an important and valuable clinical lesson which nephrologists worldwide should be made aware of, with important clinical consequences.

\section{Case Report}

We present a 55-year-old Afro-Caribbean man with a background of end-stage renal disease secondary to adult polycystic kidney disease and longstanding hypertension. He had been on renal replacement since 2006, initially on peritoneal dialysis for 2 years but converted to hemodialysis following a left nephrectomy for recurrent urosepsis. At the time of commencing cinacalcet in November 2009, he was on thrice weekly hemodialysis for 4.5 hours per session through a Fresenius ${ }^{\mathrm{TM}}$ FX100 dialyser. He was anticoagulated with the low molecular weight heparin tinzaparin, and achieved blood flow rates of $300-340 \mathrm{~mL} / \mathrm{min}$ through a left radiocephalic arteriovenous fistula that had been in use for 8 months. He was anuric, with modest interdialytic weight gains of between $1.7-2.4 \mathrm{~kg}$ between dialysis sessions, and had excellent blood pressure control off any antihypertensives. His mean pre- and post-dialysis blood pressures in November were $141 / 79$ and $133 / 78$ respectively. He did not experience any intradialytic hypotension with ultrafiltration towards his dry weight, and dialysed against a stable dialysate sodium and calcium $(1.25 \mathrm{mmol} / \mathrm{L})$.

He had no history of cardiac disease and no angina or breathlessness. An echocardiogram performed as part of a renal transplant assessment showed that the left ventricle (LV) was normal in size with normal wall thickness. Diastolic function was mildly impaired, but there was only mild global systolic dysfunction with an estimated left ventricular ejection fraction (EF) of $45 \%$.

In mid-2009 he was noted to have severe and escaping secondary hyperparathyroidism (SHPT) with an intact PTH of $1927 \mathrm{pg} / \mathrm{mL}$ (normal range $10-60 \mathrm{pg} / \mathrm{mL}$ ) and his vitamin 


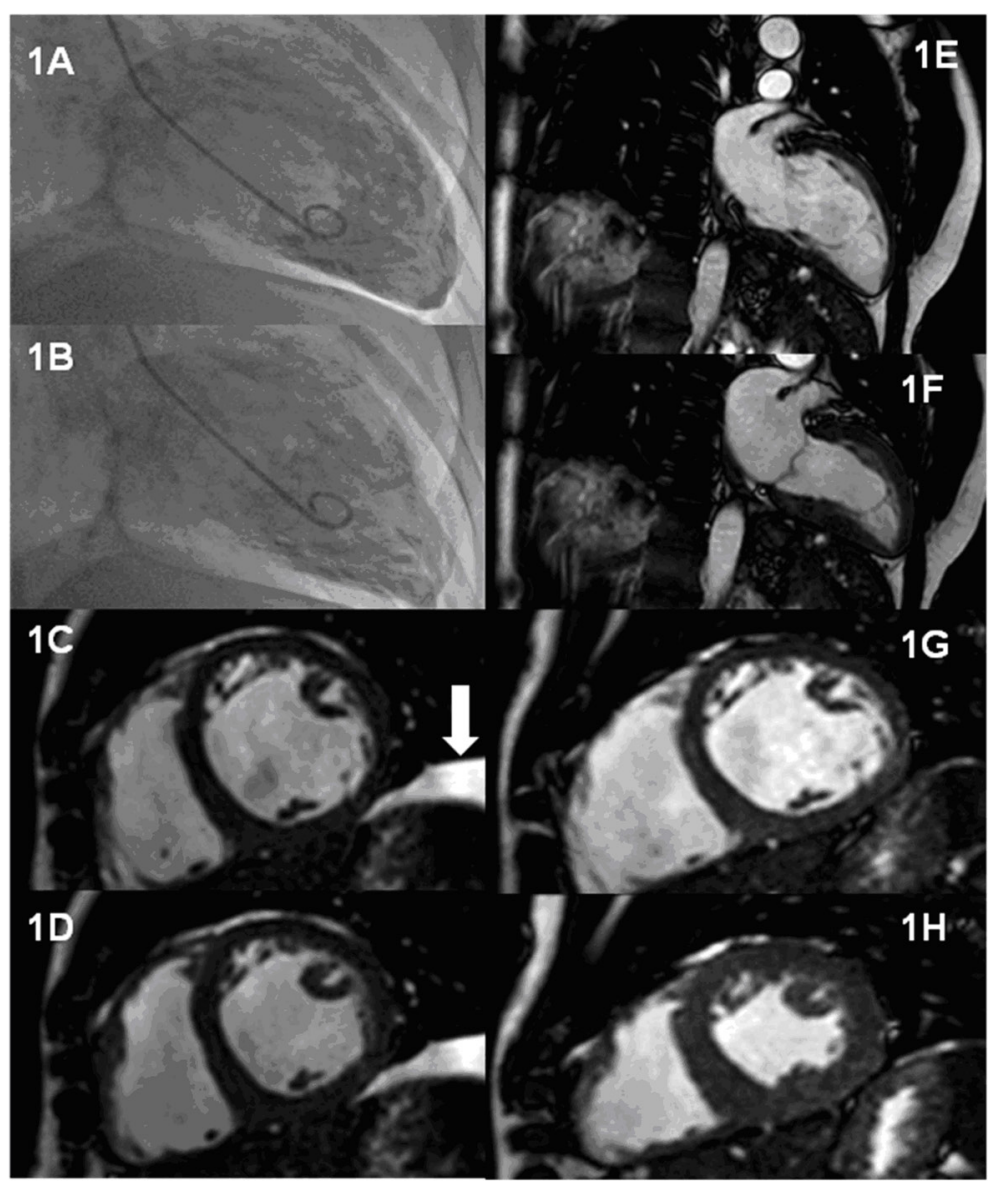

Figure 1. Serial assessment of LV function during treatment with cinacalcet and 4 months after withdrawal. Panels A-B: End-diastolic and end-systolic frames of LV angiogram performed during treatment with cinacalcet following presentation with heart failure symptoms. Panel C-D: Short axis steady state free precession (SSFP) cardiac MR images at the mid ventricular level obtained during treatment Panel C: End-diastolic frame; Panel D: End-systolic frame. The arrow indicates a left sided pleural effusion. Panels E-H: 2 chamber and mid ventricular short axis SSFP cardiac MR images obtained 4 months after discontinuation of cinacalcet. Panels $E$ and $G$ are end-diastolic frames whilst panels $F$ and $H$ are endsystolic frames.

D analogue (alfacalcidol) was increased without effect. In November his intact PTH remained above $850 \mathrm{pg} / \mathrm{mL}$, with a corrected serum calcium (cCa) of $9.6 \mathrm{mg} / \mathrm{dL}$ (NR $8.4-10$ $\mathrm{mg} / \mathrm{dL}$ ) and a phosphate of $5.48 \mathrm{mg} / \mathrm{dL}$ (NR $2.48-4.34 \mathrm{mg} /$ $\mathrm{dL}$ ) on dietary phosphate restriction, alfacalcidol $0.75 \mu \mathrm{g}$ od and sevelamer $\mathrm{HCl} 2.4 \mathrm{~g}$ tds prior to meals as a phosphate binder. He was thus commenced on cinacalcet $30 \mathrm{mg}$ od.

He was admitted in January 2010 for an expanded criteria deceased donor renal transplant, but had primary graft failure with renal vein thrombosis. He received induction immunosuppression with anti-thymocyte globulin, ciclosporin (3 doses at $7.5 \mathrm{mg} / \mathrm{kg} /$ day in divided doses), mycophenolate mofetil and prednisolone for 48 hours only. He recovered well post-operatively, and post-discharge his iPTH was persistently elevated at $792 \mathrm{pg} / \mathrm{mL}$ (cCa $9.02 \mathrm{mg} / \mathrm{dL}$, Po4 4.03 $\mathrm{mg} / \mathrm{dL}$ ). His cinacalcet was dose-increased to $60 \mathrm{mg}$ od in
January. By March 2010 he had controlled SHPT with an iPTH of $365 \mathrm{pg} / \mathrm{mL}$, aiming for 3 - 5 times the upper limit of normal as ideal in patients on dialysis [1].

Subsequently over a 4 week period he developed breathlessness and chest tightness on exertion, orthopnoea and a cough. Examination revealed a tachycardia, an elevated jugular venous pressure and bibasal crepitations in keeping with heart failure despite being dialysed to his dry weight. Chest x-ray showed new cardiomegaly. His ECG, which had been normal in January, now showed widespread $\mathrm{T}$ wave inversion across the chest leads. Coronary angiography revealed unobstructed coronary arteries with global hypokinesia and severely impaired systolic left ventricular function (Fig. 1A, B). Cardiac magnetic resonance imaging (MRI) confirmed severe biventricular dysfunction with a calculated ejection fraction of $21.5 \%$ (Fig. 1C. D). A diagnosis of dilated car- 
Table 1. Left Ventricular Volumes and Ejection Fraction Obtained From Serial Cardiac Magnetic Resonance Imaging

\begin{tabular}{lll}
\hline & During cinacalcet treatment & Post withdrawal \\
\hline LV ejection fraction $(\%)$ & 21.5 & 49.5 \\
LV stroke volume $(\mathrm{mL})$ & 53 & 130 \\
End systolic LV volume $(\mathrm{mL})$ & 197 & 133 \\
End diastolic LV volume $(\mathrm{mL})$ & 250 & 263 \\
\hline
\end{tabular}

LV, left ventricular.

diomyopathy was made and he was commenced on a betablocker and an angiotensin converting enzyme (ACE) inhibitor.

A careful clinical review revealed no cause for the development of the evolving and severe dilated cardiomyopathy. His dialysis parameters remained stable, with consistently good adequacy $(\mathrm{Kt} / \mathrm{V}$ of $>1.4)$, and modest interdialytic weight gains. Vascular access Doppler confirmed flows estimated at 1,200 mL/sec, consistent with ideal flow through a functioning brachiocephalic fistula fashioned for haemodialysis. Full virological screening was negative.

At this point cinacalcet was proposed as a potential causative agent and the drug was withdrawn. Within three months his heart failure symptoms had totally resolved. Echocardiography now showed an estimated ejection fraction of $50 \%$, the improvement in left ventricular function being confirmed by follow-up cardiac MRI (Table 1), (Fig. $1 \mathrm{E}-\mathrm{H})$. One year after stopping cinacalcet, he remains free of cardiac symptoms.

\section{Discussion}

Cinacalcet is a calcimimetic agent which acts by allosteric activation of the calcium sensing-receptor (CaR). The CaR is expressed on various cell types, including the parathyroid gland where it has been identified as the main regulator of PTH secretion. It is activated by small changes in extracellular calcium concentration and regulates bone turnover through its influence on PTH, $1 \alpha$-hydroxylation of 25-hydroxyvitamin $\mathrm{D}$, calcitonin secretion and urinary calcium excretion [2]. In clinical studies cinacalcet has been shown to lower PTH while simultaneously lowering calcium, phosphate, and calcium-phosphate product $[3,4]$. A combined post-hoc analysis of clinical events using data from 4 ( $\mathrm{n}=$ 1,184) cinacalcet phase II and III studies suggested it confers a beneficial effect on relative risk of parathyroidectomy, fracture, and hospitalization for cardiovascular complica- tions [5].

Cinacalcet is used at therapeutic doses from $30 \mathrm{mg}$ to $180 \mathrm{mg}$ od. It has several documented side effects, most commonly experienced are gastrointestinal disturbance and up to $10-15 \%$ of patients are intolerant for this reason. Transient hypocalcaemia is also recognised but is usually asymptomatic. Given the ubiquitous distribution of the $\mathrm{CaR}$ (it is present in brain tissue, the intestinal tract, vascular tissue including endothelium, smooth muscle cells, and perivascular neurons) there are relatively few site-specific side effects documented as a result of activation of $\mathrm{CaR}$ [6].

Experimental data has suggested a role for $\mathrm{CaR}$ in blood pressure modulation [7], in ischemic preconditioning in perfused mouse hearts [8], and in mediating cardiomyocyte apoptosis [9]. Given that calcium is an important second messenger throughout the cardiovascular system, and that the $\mathrm{CaR}$ is functionally expressed in atria and ventricles of rats [10], surprisingly little is known about calcium signalling through the $\mathrm{CaR}$, and even less of any cardiac effects through calcimimetic activation of these receptors.

We could find one other documented case report of a patient with known non-ischaemic dilated cardiomyopathy who was started on cinacalcet following unsuccessful surgical parathyroidectomy which precipitated severe decompensation of his cardiac failure. The symptoms resolved on withdrawal of the drug and worsened again on reintroduction. The authors suggested that the cardiac function may have adapted to the elevated serum calcium and became destabilised after reduction of the calcium levels with cinacalcet [11].

To our knowledge this is the first report of a new cardiomyopathy which developed after the initiation of cinacalcet and which resolved after withdrawal of the drug. The clear time line for the development and recovery of the severe left ventricular dysfunction in relation to the initiation and cessation, respectively, of cinacalcet implicate the drug as the causative agent. We conclude that the development of left ventricular dysfunction in patients receiving cinacalcet 
should prompt cessation of the drug.

\section{Conflict of Interest}

None.

\section{Grant}

None.

\section{References}

1. National Kidney Foundation - Kidney Disease Outcomes Quality Initiative Guidelines: Bone Metabolism and Disease in Chronic Kidney Disease. http://www. kidney.org/professionals/kdoqi/guidelines.cfm.

2. Torres PU. Cinacalcet HCl: a novel treatment for secondary hyperparathyroidism caused by chronic kidney disease. J Ren Nutr. 2006;16(3):253-258.

3. Block GA, Martin KJ, de Francisco AL, Turner SA, Avram MM, Suranyi MG, Hercz G, et al. Cinacalcet for secondary hyperparathyroidism in patients receiving hemodialysis. N Engl J Med. 2004;350(15):1516-1525.

4. Lindberg JS, Culleton B, Wong G, Borah MF, Clark RV, Shapiro WB, Roger SD, et al. Cinacalcet $\mathrm{HCl}$, an oral calcimimetic agent for the treatment of secondary hyperparathyroidism in hemodialysis and peritoneal dialy- sis: a randomized, double-blind, multicenter study. J Am Soc Nephrol. 2005;16(3):800-807.

5. Cunningham J, Danese M, Olson K, Klassen P, Chertow GM. Effects of the calcimimetic cinacalcet $\mathrm{HCl}$ on cardiovascular disease, fracture, and health-related quality of life in secondary hyperparathyroidism. Kidney Int. 2005;68(4):1793-1800.

6. Drueke TB, Ritz E. Treatment of secondary hyperparathyroidism in CKD patients with cinacalcet and/ or vitamin D derivatives. Clin J Am Soc Nephrol. 2009;4(1):234-241.

7. Smajilovic S, Yano S, Jabbari R, Tfelt-Hansen J. The calcium-sensing receptor and calcimimetics in blood pressure modulation. Br J Pharmacol. 2011;164(3):884893.

8. Sun J, Murphy E. Calcium-sensing receptor: a sensor and mediator of ischemic preconditioning in the heart. Am J Physiol Heart Circ Physiol. 2010;299(5):H13091317.

9. Sun YH, Liu MN, Li H, Shi S, Zhao YJ, Wang R, Xu $\mathrm{CQ}$. Calcium-sensing receptor induces rat neonatal ventricular cardiomyocyte apoptosis. Biochem Biophys Res Commun. 2006;350(4):942-948.

10. Smajilovic S, Tfelt-Hansen J. Calcium acts as a first messenger through the calcium-sensing receptor in the cardiovascular system. Cardiovasc Res. 2007;75(3):457467.

11. Tilling L, Colin Forfar J. Cinacalcet-associated cardiogenic shock in a patient with cardiomyopathy. Clin Ther. 2007;29(2):352-356. 